\title{
Terahertz radiation confinement using metallic resonators.
}

\author{
T. Hannotte ${ }^{1}$, M. Lavancier ${ }^{1}$, S. Mitryukovskiy ${ }^{1}, J_{-F}$ Lampin $^{1}$ and R. Peretti ${ }^{1}$ \\ ${ }^{1}$ IEMN, Univ. Lille, Villeneuve d'Ascq, France
}

\begin{abstract}
We designed, fabricated and characterized a microresonator in the terahertz range to increase light matter interaction using near-field optics. The goal of our device is to confine a $\mathrm{THz}$ electric field in a volume smaller than the diffraction limit to enhance light matter interaction with subwavelength samples. We aim at field confinement on a length scale of a few tens of nanometers.
\end{abstract}

\section{INTRODUCTION}

$\mathrm{E}$ nhancing the interaction between matter and $\mathrm{THz}$ radiation is a major requirement for the study of macromolecules, as many of them have responses in this range but are only available in small volume, far below the free space diffraction limit of a $\mathrm{THz}$ beam. Improving the quality of $\mathrm{THz}$ spectroscopy experiments has application in biology since it has been proven to be a viable approach for the characterization of biological molecular structures such as large proteins, RNA or DNA [1,2]. Light can be confined with a dielectric resonant structure such as the so called photon cages [3], or other photonic crystal cavities [4]. But reaching even smaller confinement volume compared to the wavelength requires using metallic structures. Confining $\mathrm{THz}$ radiations in a subwavelength volume to enhance light matter interaction has been done with planar metallic waveguide coupled with antennas, allowing the analysis of samples with volume of a few $\mathrm{nL}[5,6,7]$.

Another approach to surpass the diffraction limit uses near field optic to confine a non-propagating field around a nanoscale metallic object, for example the scanning tip of a SNOM (scanning near-field optical microscopy) [8]. Here we propose the use of split-ring resonators as a way to achieve the same level of confinement as a SNOM scanning tip while being compact enough to be integrated in a micro-fluidic chip.

\section{DESIGN}

A split ring resonator (SRR) is a sub wavelength resonant metallic structure analogous to a LC circuit [9]. The most basic example of SRR is a metallic loop with a small gap, where the loop is acting as an inductance, and the gap is acting as a capacitance. This kind of structure has a resonant mode with an electric field localized in the capacitive area. Since the equivalent capacity of a parallel plate capacitance is at the same time proportional to the surface area of the two plates and inversely proportional to the distance between the said plates, this capacitive area can be designed arbitrarily small while keeping the same capacity, by decreasing concordantly both variables. Same capacity implies same resonance frequency, which is convenient for tuning the design, and most importantly same energy stored in this arbitrarily small volume. This makes split ring resonators a promising way to

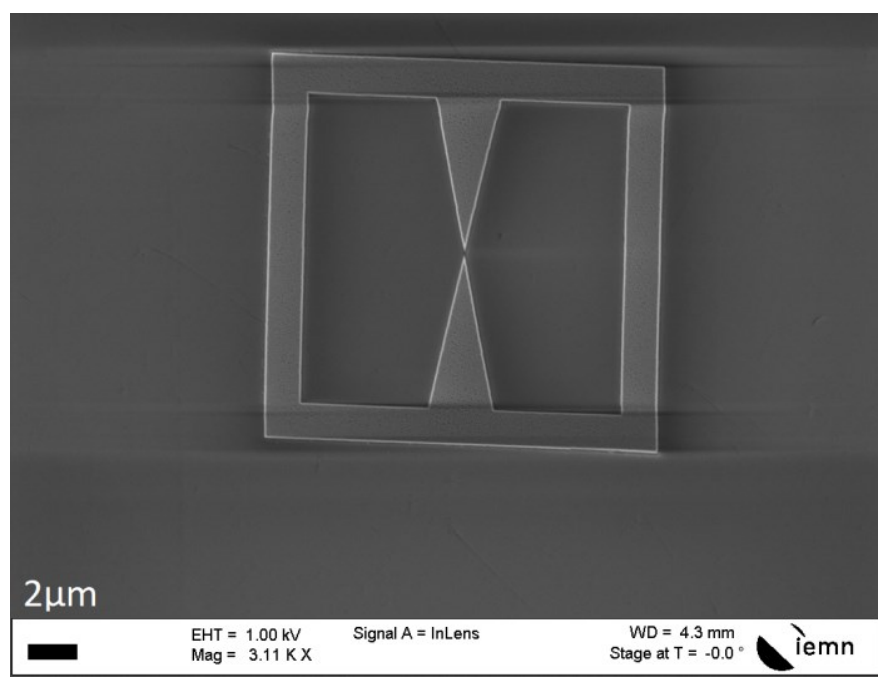

Fig. 1. SEM image of the fabricated SRR.

achieve very high spatial confinement of an electric field in the Terahertz and millimeter wave range.

The only theoretical limitation for the confinement volume arises when the size of the gap is small enough that electron cross it through quantum tunneling, making it effectively conductive and shunting the capacitance. This effect is significant only for gap sizes below $5 \mathrm{~nm}[10]$. Hence this will not be an actual limitation for enhancing light matter interaction with biological molecular structures having potential response in the $\mathrm{THz}$ range, most of which are larger than $5 \mathrm{~nm}$.

The SRR design tested here has a single gap and two inductive loops, such that the magnetic fields from both loops are cancelling each other in far field. This reduces the radiative losses of the resonator, and therefore increase its quality factor. Our SRR are 200nm thick, made out of gold on a quartz substrate. The substrate is only $150 \mu \mathrm{m}$ thick to allow microscopic observation from below.

\section{FABRICATION AND CHARACTERIZATION}

The resonators are made starting from a bare $150 \mu \mathrm{m}$ thick quartz substrate, applying a positive resist coating, and writing the SRR pattern on the coating with e-beam lithography. After resist development, $20 \mathrm{~nm}$ of Titanium and $200 \mathrm{~nm}$ of gold are then deposited on the sample by evaporation. Finally, the resist is chemically removed and the excess metal is lifted-off, leaving only the SRR on the quartz. This technology showed very good results, with excellent agreement between the wanted shape and the scanning electron microscope (SEM) observations (see figure 1). Moreover, gap size down to about $25 \mathrm{~nm}$ have been reached, which is promising for extreme field confinement experiments. 


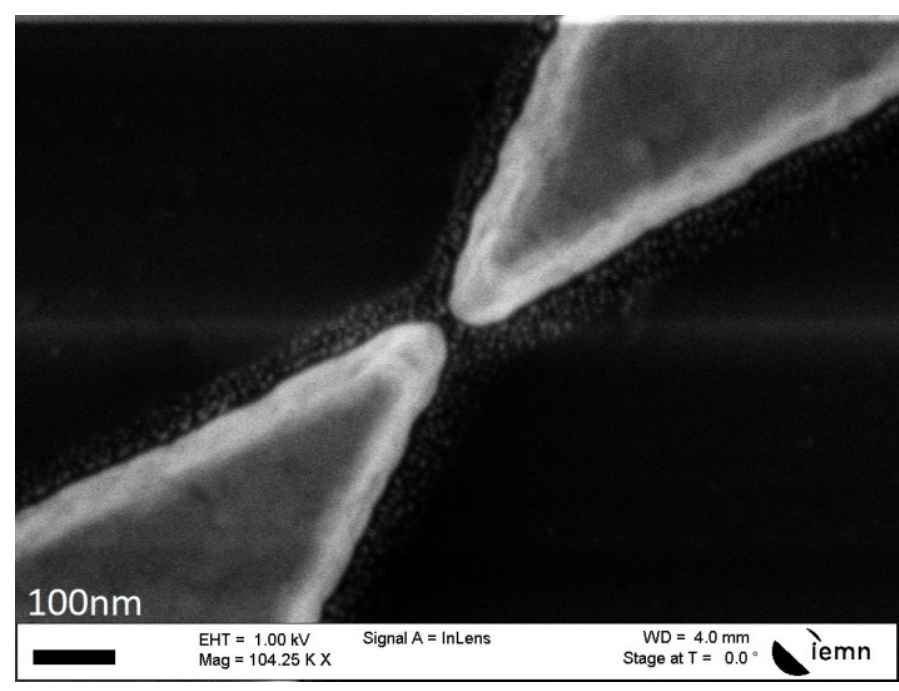

Fig. 2. SEM close view of a $\sim 25-30 \mathrm{~nm}$ gap.

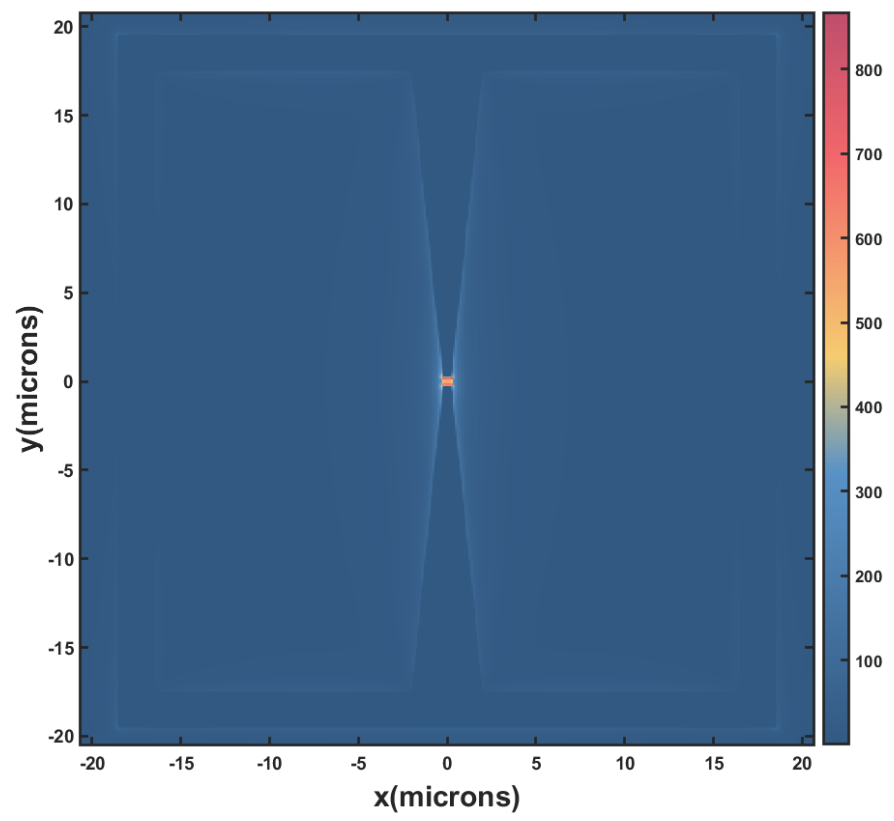

Fig. 3. Simulated electric field map at the resonance in SRR with a $500 \mathrm{~nm}$ gap. A very strong electric field is predicted in the gap.

The fabricated SRR are characterized with a THz time domain spectroscopy (TDS) setup (see figure 4). It allows to measure the temporal and spectral characteristics of the resonance mode, such as the resonance frequency and the quality factor. Those parameters are extracted directly from the temporal response with the fit@TDS software developed by our team [11].

In order to better characterize the spatial properties of the resonance, the electric field at different point around the resonator will be measured using a $\mathrm{THz}$ SNOM. This will allow us to get a map of the electric field and to measure the actual confinement volume of the field at the resonance.

\section{CONCLUSION}

We have gathered all the elements needed to study the limit of electric field confinement in a split ring resonator, potentially reaching a few tens of nanometer scale at $2.5 \mathrm{THz}$, which

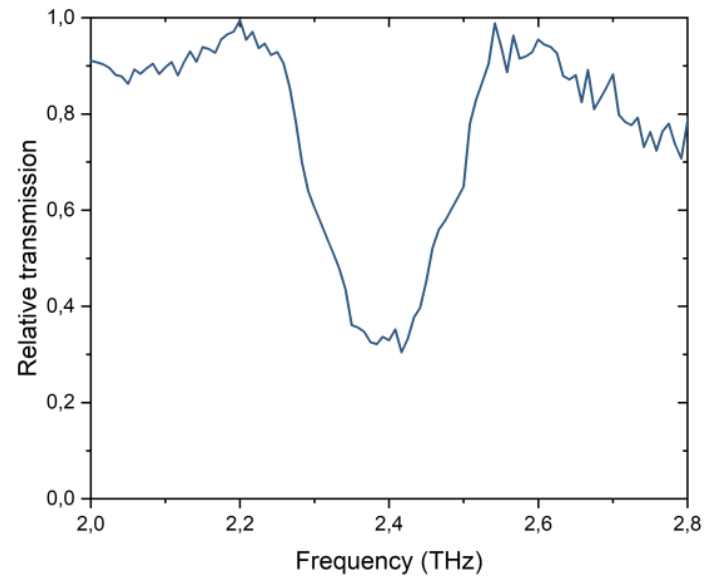

Fig. 4. Measured transmission spectrum of an array of resonator with TDS, relative to the transmission of a bare quartz substrate.

represent a relative length of $\lambda / 4000$. Such a confinement needs to be further characterized, but is very promising to reach high enhancement of light matter interaction at very small scale such as predicted in numerical simulation (FDTD software from Lumerical, see figure 3). This will allow the derivation of the Purcell factor and subsequent conclusion of possible strong coupling or other quantum effects.

This work will constitute a powerful tool for the study of the susceptibility of nanometer sized elements with a terahertz response, such as many biological samples like viruses and proteins. In addition, it could be extended to more fundamental experiments aiming at exploring quantum effects in the terahertz range.

\section{REFERENCES}

[1]. T. Globus, et al, "THz-Spectroscopy of Biological Molecules". Journal of Biological Physics (2003) 29: 89.

[2]. E. Castro-Camus et al, "Conformational changes of photoactive yellow protein monitored by terahertz spectroscopy". Chemical Physics Letters 455.4-6 (2008), pp. 289-292.

[3]. C. Sieutat et al, "Strong confinement of light in low index materials: the Photon Cage". Opt. Express 21, 20015-20022 (2013).

[4]. N. Descharmes et al, "Observation of Backaction and Self-induces Trapping in a Planar Hollow Photonic Crystal Cavity". Physical Review Letters, American Physical Society (APS), 2013, 110

[5]. R. Peretti, et al, "Broadband terahertz lightmatter interaction enhancement for precise spectroscopy of thin films and micro-samples". Photonics, 5(2):11, may 2018.

[6]. S. Mitryukovskiy et al. "Shining the Light to Terahertz Spectroscopy of nL-Volume Biological Samples". Conference on Lasers and Electro-Optics. OSA, 2019

[7]. R. Peretti et al, "Device for Light-Matter Interaction Enhancement in the Full THz Range for Precise Spectroscopy of Small Volume Samples". $201843^{\text {rd }}$ International Conference on Infrared, Millimeter, and Terahertz Waves (IRMMW-THz), IEEE (2018).

[8]. S. Mastel, et al, "Terahertz nanofocusing with cantilevered terahertzresonant antenna tips". Nano Letters, 17(11):6526-6533, 2017.

[9]. W. Froncisz et al. "The loop-gap resonator: a new microwave lumped circuit ESR sample structure". Journal of Magnetic Resonance (1969) 47.3 pp. 515-521 (1982)

[10]. J. A. Scholl et al, "Observationof Quantum Tunneling between Two Plasmonic Nanoparticles". Nano Letters, American Chemical Society (ACS), 2013, 13, 564-569

[11]. R. Peretti, et al, "THz-TDS time trace analysis for physical parameters extraction", IEEE transactions on Terahertz Science and Technology (2018). Source code: https://github.com/THzbiophotonics/Fit-TDS 